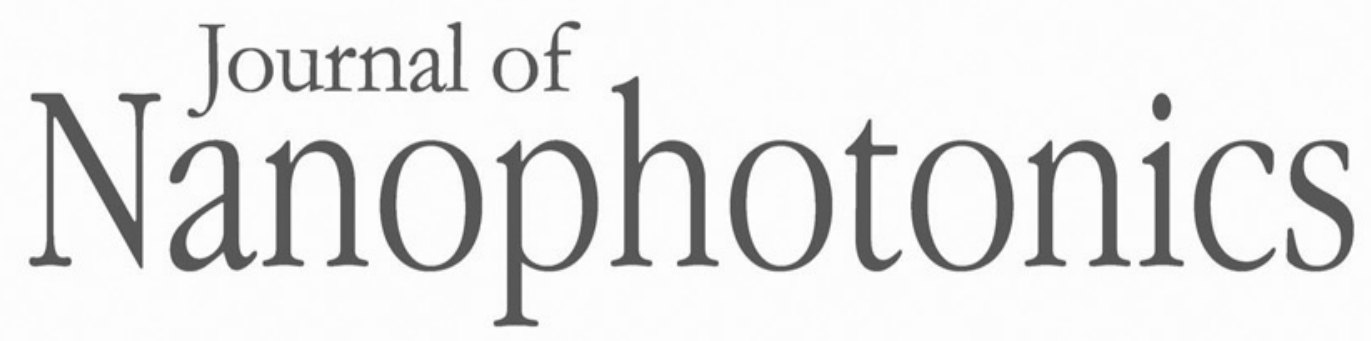

Nanophotonics.SPIEDigitalLibrary.org

\title{
Surface-enhanced Raman scattering detection of toluene and dichlorobenzene vapors using 1- propanethiol-linked Ag nanoparticles
}

NoSoung Myoung Hyung Keun Yoo In-Wook Hwang 


\title{
Surface-enhanced Raman scattering detection of toluene and dichlorobenzene vapors using 1-propanethiol-linked Ag nanoparticles
}

\author{
NoSoung Myoung,* Hyung Keun Yoo, and In-Wook Hwang* \\ Advanced Photonics Research Institute, Gwangju Institute of Science and Technology, \\ Gwangju 500-712, Republic of Korea
}

\begin{abstract}
We have detected the surface-enhanced Raman scattering (SERS) signals of toluene and 1,2-dichlorobenzene (ODCB) vapors at parts per million concentrations using 1-propanethiol-linked Ag nanoparticles and a fiber optics-coupled Raman spectrograph. Calibration curves were constructed for each detector by plotting Raman band intensity ratios of toluene and ODCB with respect to that of 1-propanethiol as a function of vapor concentration. The detectors showed effective vapor sensing ranges of 0.6 to 600 and 10 to $600 \mathrm{ppm}$ for toluene and ODCB, respectively, following vapor adsorption to the SERS hot spots. We provide evidence for the facility of the SERS detection system for instantaneously discriminating and quantifying respective vapors from a composite vapor. () The Authors. Published by SPIE under a Creative Commons Attribution 3.0 Unported License. Distribution or reproduction of this work in whole or in part requires full attribution of the original publication, including its DOI. [DOI: 10.1117/1.JNP.8.083083]
\end{abstract}

Keywords: Raman spectroscopy; surface-enhanced Raman scattering; Ag nanoparticle; gas sensor; volatile organic compounds sensing.

Paper 14032 received Mar. 20, 2014; revised manuscript received May 20, 2014; accepted for publication May 27, 2014; published online Jun. 24, 2014.

\section{Introduction}

The development of surface-enhanced Raman scattering (SERS)-based detectors has been inspired by a growing demand for high sensitivity and facile detection of signals corresponding to organic molecules in the fingerprint regime. Identification and quantification of molecules are of importance, particularly in practical fields such as environmental and industrial monitoring of toxic vapors, biological sensing, ${ }^{1-3}$ and medical applications. ${ }^{4}$ Because the vapor sensor using the SERS is influenced by both the SERS effect and gas adsorption, it is of importance to find a method to efficiently adsorb vapor samples to a SERS "hot spot." Several surface modification methods for deducing organic molecule adsorption to SERS hot spots have been attempted. Goncalves et al. ${ }^{5}$ recently modified the surfaces of triangular Ag nanostructures with thiol units for detecting organic dyes. They observed intense Raman signals from the dyes adsorbed to the thiol holder units linked to Ag nanostructures, as measured by confocal microscopy. The regions showing strong near-field enhancements i.e., sharp edges of the silver triangular particles, played roles of SERS hot-spots. Deschaines et al. ${ }^{6}$ detected SERS signals from aqueous organic samples adsorbed to alkythiol units linked to Ag foils. According to this study, 1-propanethiol, which was covalently linked to Ag foil, was more effective for sensing aromatic molecules compared to 1dodecanethiol and $p$-cresolthiol. The authors demonstrated that the superior property of shorter 1-propanethiol in an alkane chain length resulted in an increased SERS effect, which was enabled by closer adsorption of sample molecules to SERS hot spots.

In this report, we focus on the detection of vapor-phase toluene and 1,2-dichlorobenzene (ODCB) by using 1-propanethiol-linked Ag nanoparticles (NPs), which have been optimized to show intense SERS signals. Toluene and ODCB vapors are chosen as samples because they are typical industrial organic compounds known as hazardous substances, which are regulated by OSHA. ${ }^{7}$ Toluene and ODCB vapors are found to be efficiently adsorbed onto SERS hot spots of

*Address all correspondence to: NoSoung Myoung, E-mail: nsmyoung@gist.ac.kr or In-Wook Hwang, E-mail: hwangiw@gist.ac.kr 
the NPs, particularly because of hydrophobic interactions at the interface of the aromatic units of the vapors and NP-linked propane units. From analysis of the SERS signals obtained for toluene and ODCB vapors, we demonstrate that the detectability of the vapors is significantly influenced by vapor adsorption to the SERS hot spots rather than the degree of SERS effects.

\section{Materials and Methods}

The SERS substrates were prepared using previously reported wet chemistry processes. ${ }^{8}$ A silicon dioxide $\left(\mathrm{SiO}_{2}\right)$ layer with a thickness of $\sim 500 \mu \mathrm{m}$ was deposited onto a silicon wafer by a chemical vapor deposition method. The substrate was dipped into a solution composed of $\mathrm{H}_{2} \mathrm{SO}_{4}: \mathrm{H}_{2} \mathrm{O}_{2}(3: 1)$ at $65^{\circ} \mathrm{C}$ for $30 \mathrm{~min}$ in order to attach hydrophilic $\mathrm{OH}$ groups onto the $\mathrm{SiO}_{2}$ layer, followed by dipping into a solution of (3-aminopropyl)trimethoxysilane (APTMS):ethanol (1:9) at room temperature (RT) for $2 \mathrm{~h}$ to functionalize the $\mathrm{SiO}_{2}$ surface with amine moieties. A single layer of Ag NPs was fabricated by dipping the substrate into Ag NP inks (average NP diameter of $\sim 100 \mathrm{~nm}$, Sigma-Aldrich, Milwaukee, Wisconsin) diluted with deionized water by a factor of 1000 (12 h at RT). Multiple layers of Ag NPs were made by first dipping the singlelayered substrate into a solution composed of 1-mL APTMS, 10-mL ethanol, and 0.1-mL $1 \mathrm{M}$ $\mathrm{HCl}(2 \mathrm{~h}$ at RT) to activate the Ag NP surfaces, followed by dipping into Ag inks; these processes were repeated to further stack Ag NPs. 1-Propanethiols were finally linked to Ag NPs by dipping the multiple-layered substrate into an aqueous 1-M 1-propanethiol solution ( $24 \mathrm{~h}$ at RT).

Raman spectra were recorded with a fiber optics-coupled Raman spectrograph comprised of a diode laser operating at $785 \mathrm{~nm}$ and with $\sim 15 \mathrm{~mW}$, an optical fiber bundle for photoexciting the samples and collecting the Raman-scattered signals (EmVision LLC., Florida), band- and long-pass filters, a monochromator, and a thermoelectrically cooled charge-coupled device (CCD) detector [Fig. 1(a)]. The 1200-g/mm grating and CCD pixel size of $26 \mu \mathrm{m}$ yield a spectral dispersion of $2.6 \mathrm{~cm}^{-1} /$ pixel. For minimizing the thermal heating effect on the samples and the Raman spectra, we used a low laser excitation power $(15 \mathrm{~mW})$ and a very short data acquisition time (1 s). The optical-fiber bundle, consisting of one core excitation fiber of $400 \mu \mathrm{m}$ in diameter and seven surrounding collection fibers, may also show better detection sensitivity toward Raman-scattered signals [Fig. 1(b)]. Raman spectra of the vapors were measured by evaporating a calculated volume of toluene or ODCB solvent added to a capped 1000-mL flask containing the SERS substrate and coupled to the optical-fiber bundle [Fig. 1(a)]. An equilibration time of $30 \mathrm{~min}$ between each measurement was allowed to achieve vapor diffusion equilibrium inside the flask. Raman spectra were instantaneously recorded by summing 10 data points obtained at each collection time (1 s). For the analysis of Raman spectra, we subtracted baselines from raw spectral data by using the fifth order modified polynomial curve fitting procedure with Wolfram Mathemtica 9.0 based on the C. A. Liber group. ${ }^{9}$

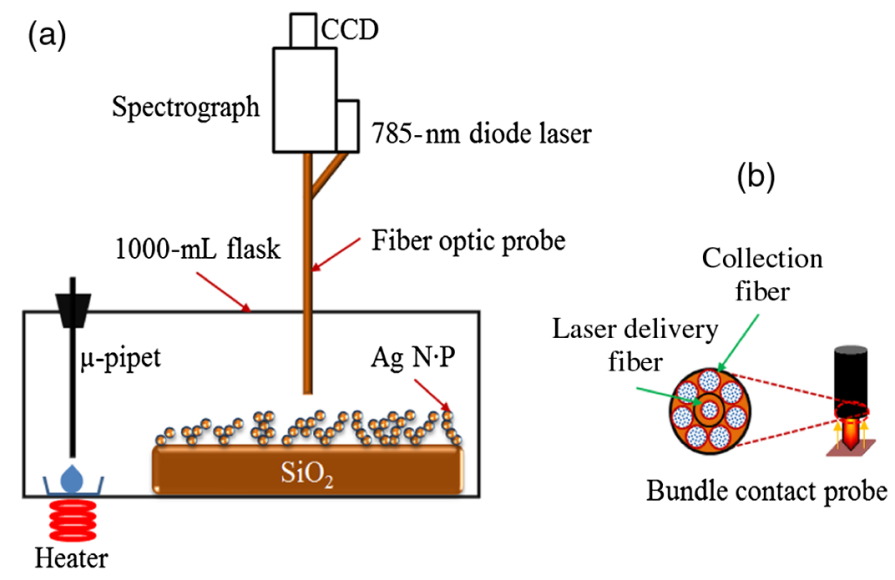

Fig. 1 Schematic view of surface-enhanced Raman scattering (SERS) measurement of organic vapors. A $1000-\mathrm{mL}$ volumetric flask (a) and an optical-fiber bundle comprising one core excitation fiber and seven surrounding collection fibers (b) are used. The excitation wavelength and intensity was $785 \mathrm{~nm}$ and $15 \mathrm{~mW}$, respectively. 


\section{Results and Discussion}

The scanning electron microscope (SEM) images shown in Fig. 2 demonstrate that the Ag NPs were systematically deposited onto the $\mathrm{SiO}_{2}$ substrates by wet chemistry processes. In this study, we used SERS substrates fabricated by Ag NPs applied over four coating cycles (average NP diameter of $\sim 100 \mathrm{~nm}$ ) because the obtained coating yielded the highest SERS signal intensity; further deposition of NPs was found to result in a reduced SERS signal intensity because of a limitation in the number of SERS hot spots. ${ }^{10}$ The Ag NPs were modified with 1-propanethiol molecules for adsorption and detection of hydrophobic toluene and ODCB vapors. The propane units linked to Ag NPs are thought to interact with the aromatic units of organic vapors via hydrophobic van der Waals interactions. This interaction leads to a linear behavior in the SERS signal intensity enhancement as a function of vapor concentration, as will be noted.

Bare and 1-propanethiol-linked Ag NPs deposited onto the $\mathrm{SiO}_{2}$ substrates were examined for toluene vapor detection (black and blue lines of Fig. 3). The Raman spectrum of a SERS substrate (i.e., 1-propanethiol-linked Ag NPs that were not exposed to toluene vapor) was also plotted for comparison (red line of Fig. 3). When exposed to toluene vapor (at $600 \mathrm{ppm}$ ), bare Ag NPs did not show remarkable Raman-scattered signals in the regime of 600 to $1400 \mathrm{~cm}^{-1}$, while
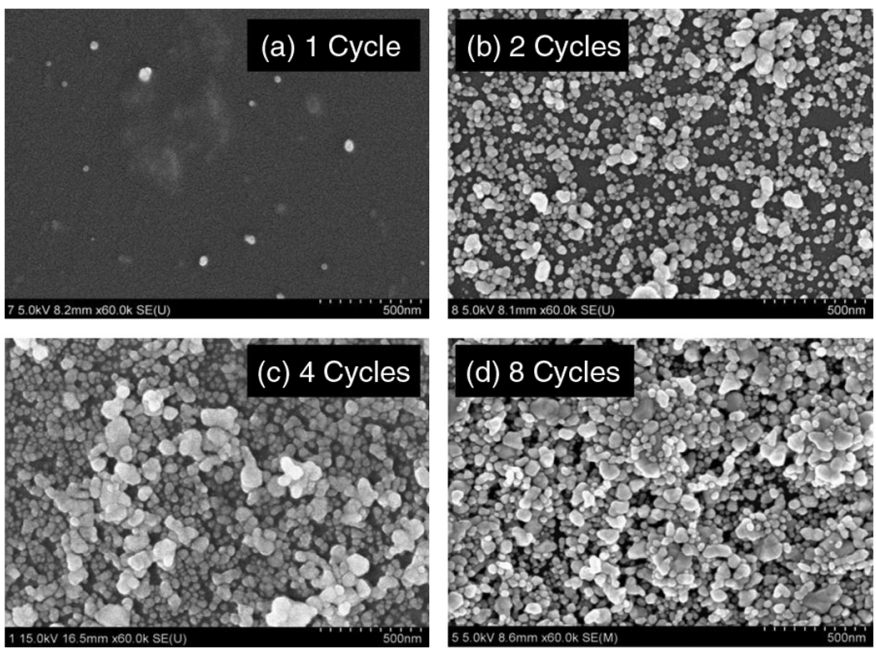

Fig. 2 Scanning electron microscope (SEM) images for Ag NPs prepared by (a) one, (b) two, (c) four, and (d) eight deposition cycles.

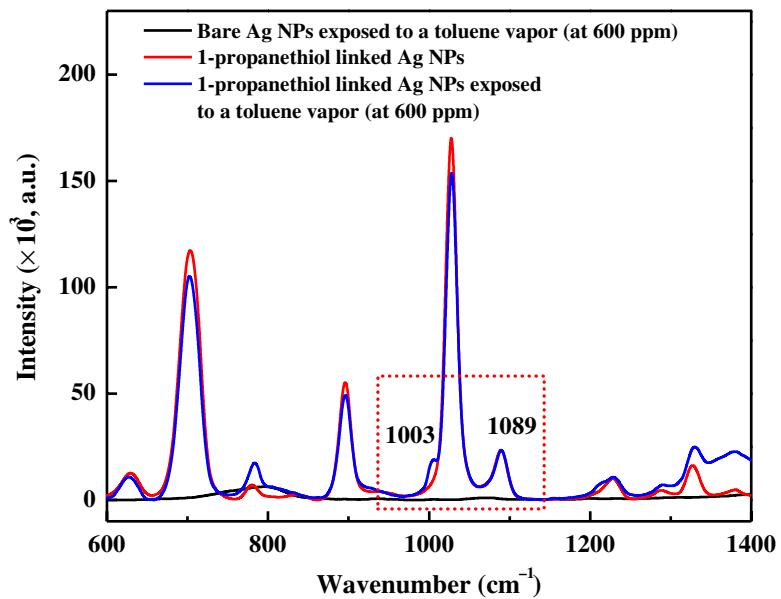

Fig. 3 Raman spectra for bare Ag NPs exposed to a toluene vapor (black, at 600 ppm), 1-propanethiol linked Ag NPs (red), and 1-propanethil linked Ag NPs exposed to a toluene vapor (blue, at $600 \mathrm{ppm}$ ). Baselines were consistently subtracted from the Raman spectra using a polynomial function. 
1-propanethiol-linked Ag NPs exhibited a summation of the Raman-scattered signals from 1-propanethiol and toluene, which is indicative of efficient adsorption of toluene vapors to the SERS hot spots of NPs. Note that the Raman band at $1003 \mathrm{~cm}^{-1}$ (corresponding to $\mathrm{C}-\mathrm{C}$ stretching mode of toluene ${ }^{11}$ ) was amplified as the toluene vapor was adsorbed, while that at $1089 \mathrm{~cm}^{-1}$ (corresponding to the $\mathrm{CH}_{3}$ rocking mode of 1-propanethiol ${ }^{10,12}$ ) showed only a negligible change in intensity. We used the Raman bands at 1003 and $1089 \mathrm{~cm}^{-1}$ as sample and reference marker bands, respectively, for calibrating the unknown concentration of toluene vapor samples. The Raman band at $1130 \mathrm{~cm}^{-1}$ (corresponding to the $\mathrm{C}-\mathrm{H}$ bending mode of ODCB ${ }^{13}$ ) was also used as a sample band.

Figures 4(a) and 4(b) show SERS signals for toluene and ODCB vapors, respectively, plotted as a function of vapor concentration in the range of 10 to $800 \mathrm{ppm}$. By increasing the vapor concentration, Raman bands at 1003 and $1130 \mathrm{~cm}^{-1}$ (corresponding to toluene and ODCB, respectively) systematically increased in intensity. Calibration for respective SERS substrates and sample vapors was performed by plotting the intensity ratios of Raman bands corresponding to the sample (at 1003 and $1130 \mathrm{~cm}^{-1}$ ) and reference (at $1089 \mathrm{~cm}^{-1}$ ) as a function of vapor concentration [Figs. 4(c) and 4(d)]; this procedure was performed in order to eliminate spotto-spot dependence of the SERS substrate upon the Raman signal sensitivity.

Note that in Fig. 4(c), the data for both toluene and ODCB vapors clearly showed intensity enhancements in the range of 10 to $600 \mathrm{ppm}$, while they were saturated at $>600 \mathrm{ppm}$. This result is indicative of the upper detection limit of toluene and ODCB vapors as $\sim 600 \mathrm{ppm}$, probably because of saturation in a vapor adsorption capacitance. The lowest vapor detection limit was determined by measuring sample vapors with very low concentrations of $<1 \mathrm{ppm}$ [Fig. 4(d)]. The data for a toluene vapor sample at $0.6 \mathrm{ppm}$ exhibited an intensity enhancement, compared to that at $0 \mathrm{ppm}$, whereas that for ODCB at $0.6 \mathrm{ppm}$ did not show such an enhancement [Fig. 4(d)]. This result is indicative of the lowest detection limits of toluene and ODCB as $\sim 0.6$ and $>0.6 \mathrm{ppm}$, respectively; in our measurement, the lowest detection limit of ODCB was estimated
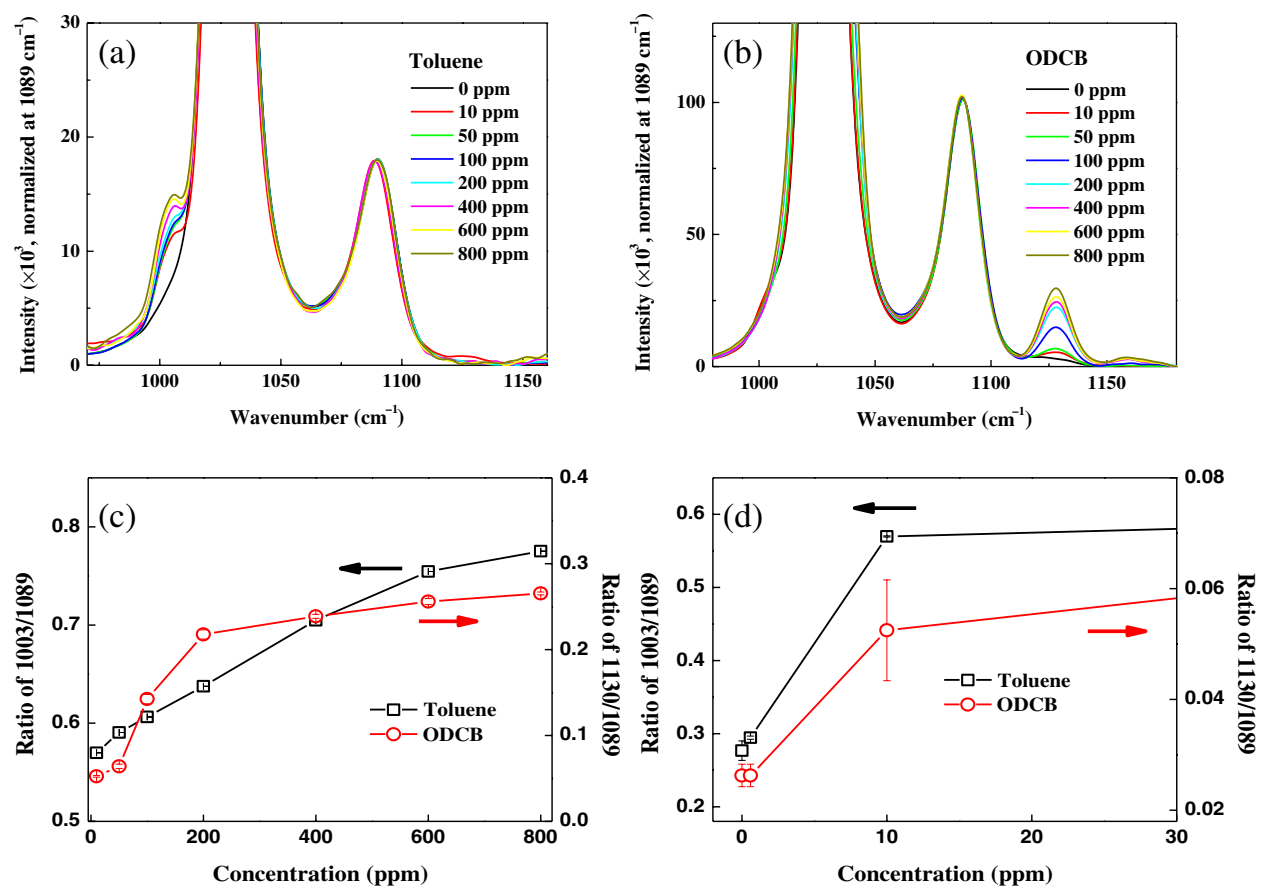

Fig. 4 Raman spectra for (a) toluene and (b) ODCB vapors plotted as a function of vapor concentration in the range of 10 to $800 \mathrm{ppm}$. The spectra were plotted after baseline subtraction and intensity normalization at $1089 \mathrm{~cm}^{-1}$. (c) Calibration curves for toluene (black) and ODCB (red) vapors, which are obtained by plotting Raman band intensity ratios of baseline-subtracted Raman spectra corresponding to the sample and the reference in the range of 10 to $800 \mathrm{ppm}$; error bars indicate standard deviation from the experiment data. (d) Calibration curves for toluene (black) and ODCB (red) vapors in the low concentration regime of 0 to $30 \mathrm{ppm}$. 
to be $\sim 10$ ppm, as denoted in Figs. 4(b) and 4(d). Because consistent experimental conditions were used for both vapors, the better detection limit of toluene $(0.6 \mathrm{ppm})$ over that of ODCB $(10 \mathrm{ppm})$ may result from the higher affinity of toluene to the propane unit rather than different SERS effects. Note that the solvent polarity indices of toluene and ODCB are 2.4 and 2.7, respectively, with respect to 0 for $n$-hexane. ${ }^{14}$ Permissible exposure limits for toluene and ODCB vapors in general industry are reported to be 200 and $50 \mathrm{ppm}$, respectively, for a short-term exposure limit. ${ }^{15}$

In an attempt to verify the efficiency of simultaneous detection of both vapors, we measured the SERS signals from a composite vapor comprised of toluene (at $200 \mathrm{ppm}$ ) and ODCB (at 200 ppm) [Fig. 5(a)]. Raman band intensity ratios (0.67 and 0.18) characterized for the respective toluene and ODCB component vapors were in good agreement with the calibrated plots for the 200 ppm vapors [Fig. 4(c)]. This result is indicative of the ability of the present SERS detection system to discriminate and quantify respective vapors.

We finally performed a real-time detection of flowing toluene vapor at RT [Fig. 5(b)]. This measurement was enabled by fast data acquisition using the SERS detector, a sample chamber $\left(1 \times 2 \times 2 \mathrm{~m}^{3}\right)$ equipped with inlet and outlet valves, and a homemade program (Visual Basic) for real-time reading of Raman band intensities. The SERS detector was found to read the same concentration values as those obtained using a commercialized conductivity detector (Model FP700, Detcon Inc., The Woodland, Texas), when two detectors were placed at very close positions in the sample chamber. This result is indicative of the potential of the developed SERS detection system to be utilized in a real field application. As the toluene vapor was injected, the SERS detector exhibited a continuous signal increment at a low concentration regime of $<20 \mathrm{ppm}$ or at
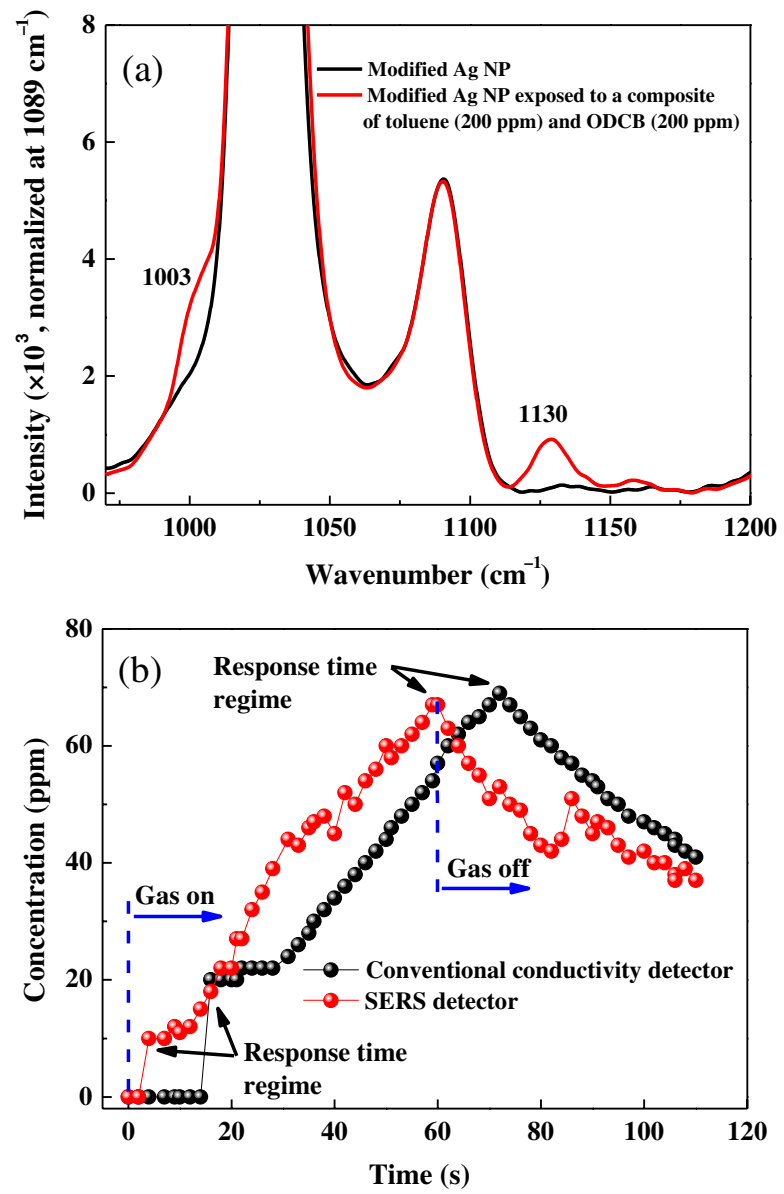

Fig. 5 (a) Raman spectra for a composite vapor comprising toluene (at $200 \mathrm{ppm}$ ) and ODCB (at 200 ppm) (red) and for a reference SERS substrate (black). (b) Data for a real-time monitoring of flowing toluene vapor, obtained by SERS detector (red) and conventional conductivity detector (black). 
a fast time regime of $<12 \mathrm{~s}$, whereas the conductivity detector did not show any responses at this regime, indicative of better sensitivity and faster response of the developed SERS detector than those for the conventional conductivity detector. In addition to these advantages, a SERS detector with the capability to discriminate individual component vapors from a vapor complex is of importance [Fig. 5(a)].

\section{Conclusions}

From SERS spectral analysis of organic vapors adsorbed onto SERS hot spots, we demonstrate that the SERS substrates consisting of 1-propanethiol-modified Ag NPs show effective vapor sensing ranges of 0.6 to 600 and 10 to $600 \mathrm{ppm}$ for toluene and ODCB, respectively. The upper detection limit of the vapors ( $600 \mathrm{ppm})$ was found to be principally determined by the capacitance of vapor adsorption, whereas the lowest detection limit $(0.6$ or $10 \mathrm{ppm})$ was influenced by intermolecular interactions at the interface of the sample (toluene and ODCB) and reference (propane) units. Using the SERS detector, we successfully performed a real-time detection of flowing toluene vapor and a detection of a composite vapor comprised of toluene and ODCB.

\section{Acknowledgments}

This research was supported by the APRI Research Program of GIST from the Basic Science Research Program, the Eco-Innovation project [ARQ20114000114 (401-112-014)] funded by the Korea Ministry of Environment, and Basic Science Research Program through the National Research Foundation of Korea (NRF) funded by the Ministry of Education (2011-0006563 and 2013R1A1A2058746).

\section{References}

1. A. J. Haes et al., "Nanoscale optical biosensor: short range distance dependence of the localized surface plasmon resonance of noble metal nanoparticles," J. Phys. Chem. B 108(22), 6961-6968 (2004).

2. N. Anderson et al., "Nanoscale vibrational analysis of single-walled carbon nanotubes," $J$. Am. Chem. Soc. 127(8), 2533-2537 (2005).

3. Y. S. Huh, A. J. Chung, and D. Erickson, "Surface enhanced Raman spectroscopy and its application to molecular and cellular analysis," Microfluid. Nanofluid. 6(3), 285-297 (2009).

4. G. Zhen et al., "Multiplexed electrical detection of cancer markers with nanowire sensor arrays," Nat. Biotechnol. 23, 1294-1301 (2005).

5. M. R. Goncalves, F. Enderle, and O. Marti, "Surface-enhanced Raman spectroscopy of dye and thiol molecules adsorbed on triangular silver nanostructures: a study of near-field enhancement, localization of hot-spots, and passivation of adsorbed carbonaceous species,' J. Nanotechnol. 2012, 173273 (2012).

6. T. O. Deschaines and K. T. Carron, "Stability and surface uniformity of selected thiol-coated SERS surfaces,” Appl. Spectrosc. 51(9), 1355-1359 (1997).

7. W.-L. Zhai et al., "Multiple depositions of Ag nanoparticles on chemically modified agarose films for surface-enhanced Raman spectroscopy," Nanoscale 4, 137-142 (2012).

8. U.S. Department of Labor, "Occupational Safety \& Health Administration," Regulations (Standard- 29 CFR 1910.1000 Table Z-1, 2) (2012).

9. C. A. Liber and A. M. Jansen, "Automated method for subtraction of fluorescence from biological Raman spectra," Appl. Spectrosc. 57(11), 1363-1367 (2003).

10. T. Torgrimsen and P. Klaeboe, "The vibrational spectra and the stable conformers of 1-propanethiol," Acta Chim. Scand. 24, 1139-1144 (1970).

11. H. F. Hameka and J. O. Jensen, "Theoretical studies of the methyl rotational barrier in toluene," Theochem. J. Mol. Struct. 362(3), 325-330 (1996).

12. R. E. Pennington et al., "The chemical thermodynamic properties and rotational tautomerism of 1-propanethiol," J. Am. Chem. Soc. 78(14), 3266-3272 (1956). 
Myoung, Yoo, and Hwang: Surface-enhanced Raman scattering detection of toluene and...

13. T. Anno and I. Matubara, "Analysis of the near ultraviolet absorption spectra of ortho- and para-dichlorobenzene vapors," J. Chem. Phys. 23, 796 (1955).

14. A. F. M. Barton, CRC Handbook of Solubility Parameters and Other Cohesion Parameters, 2nd ed., CRC Press LLC, Florida (1991).

15. U.S. Department of Labor, "Occupational Safety \& Health Administration," Regulations (Standard- 29 CFR 1910.1000 Table Z-1, 2) (2012).

NoSoung Myoung received his $\mathrm{PhD}$ degree in physics for his work on the development of midinfrared solid-state lasers from University of Alabama at Birmingham, in 2011. From 2011 to 2013, he worked as a senior research engineer in Samsung Display Research Institute, South Korea. Since 2013, he has been a research fellow in the APRI at Gwangju Institute of Science and Technology (GIST), South Korea. His main fields of interest include the application of laser spectroscopy for surface-enhanced Raman spectroscopy (SERS), and the development and characterization of novel laser systems based on semiconductor materials with transitional metals.

Hyung Keun Yoo is a postdoctoral researcher of the Advanced Photonics Research Institute at Gwangju Institute of Science and Technology (GIST), Gwangju, Republic of Korea. He has investigated terahertz wave spectroscopy and application based on carrier dynamics in optically excited states of organic semiconductors. He received his MS degree in physics in 2006, and his $\mathrm{PhD}$ degree in 2012 from Sogang University, South Korea.

In-Wook Hwang is a senior research scientist of the Advanced Photonics Research Institute at Gwangju Institute of Science and Technology (GIST), South Korea. His research fields are ultrafast laser spectroscopy and organic solar cells. He received his $\mathrm{PhD}$ degree in chemistry at Yonsei University (2002). In the period from 2002 to 2009, he worked as a researcher in Yonsei University, UCSB, and GIST, characterizing ultrafast energy and electron dynamics in various organic systems (porphyrin arrays, liquid crystals, and semiconducting polymers) and fabricating organic solar cells. 